\title{
Electromagnetic Imaging of Three-Dimensional Dielectric Objects with Newton Minimization
}

\author{
Aslan Etminan, Alireza Sadeghi, and Levent Gürel \\ Bilkent University Computational Electromagnetics Research Center (BiLCEM), Ankara, TR-06800, Turkey \\ lgurel@gmail.com
}

\begin{abstract}
We present a general framework for detecting the shape and electrical properties of unknown objects by using the Newton minimization approach for solving inverse-scattering problems. This procedure is performed by evolving an initialguess object iteratively until the cost function decreases to a desired value. Rapid convergence of this method is demonstrated by some numerical results.
\end{abstract}

\section{INTRODUCTION}

Due to the nondestructive penetration property of electromagnetic waves, microwave imaging has been a suitable and attractive technique for geophysical probing, target identification, and medical imaging. The main goals of imaging are to detect the location, shape, and electromagnetic material properties of an unknown object. Despite its importance, simultaneous determination of all of these parameters successfully is quite rare. One of the most common biomedical applications is breast cancer detection, and its goal is to find the location of the tumor. One approach for microwave imaging is using finite-difference time-domain simulators and then employing radar and signal processing techniques [1]. Another approach is to solve an inverse-scattering problem in the frequency domain [2]. This method has a wide range of applications, e.g., underground resource detection [3]. If the investigated problem has such a geometry that is invariant in one direction, the three-dimensional (3-D) inverse-scattering problem can be transformed into a two-dimensional problem. Nevertheless, it is not an easy task to determine simultaneously both the 3-D shape and the material properties of an unknown object. In this paper, to obtain the shape and electrical properties of an unknown 3-D object, we solve an inverse-scattering problem by minimizing a cost function [4] and by utilizing a forward solver based on the multilevel fast multipole algorithm [5].

\section{The Cost Function and Its Minimization}

The general idea of a large group of minimization methods is to start with an initial guess of the parameters of the function that should be minimized and update this initial guess iteratively in such a way that we ultimately obtain a set of these parameters, which minimizes the target function. In this work, we aim to detect the shape and material properties of unknown objects simultaneously. For this goal, we start with an initial-guess object and choose a set of initial values for the material properties of the initial-guess object. By illuminating the objects and collecting the scattered fields, we achieve two sets of measurements. After defining the cost function as the total mismatch between these two sets, our microwave imaging problem can be transformed into the minimization of the cost function. The cost function can be written as

$$
C(\boldsymbol{x})=\sum_{i=1}^{M}\left|e_{i}(\boldsymbol{x})\right|^{2}=\sum_{i=1}^{M}\left|S_{i}(\boldsymbol{x})-m_{i}\right|^{2},
$$

where $e_{j}(\boldsymbol{x})=S_{j}(\boldsymbol{x})-m_{j}$ is the mismatch between the $j$ th measurements collected from the evolving initial-guess object $S_{j}(\boldsymbol{x})$ and the target $m_{j}$, and $M$ is the number of measurements. In [2], where we only reconstructed the shape of targets, we prepared a modeling vector $\boldsymbol{x}$, which contained the locations of nodes on the surface of the evolving object, and updated this vector by using the steepest-descent method such that the cost function decreased iteratively. In this work, because we desire to obtain the electromagnetic material properties of the target, we should consider these electromagnetic properties in modeling the evolving initialguess object. Therefore, we define the material vector of the evolving object at the $k$ th iteration in the form

$$
\boldsymbol{x}_{k}^{m}=\left[\begin{array}{c}
\sigma_{k} \\
\epsilon_{k}
\end{array}\right],
$$

where $\sigma_{k}$ and $\epsilon_{k}$ are the conductivity and permittivity of the evolving initial-guess object at the $k$ th iteration. Similar to the minimization method that we used in [2] to reconstruct the shape of the target, we update the material vector elements iteratively to obtain the material properties of the real object. We update the material vector by

$$
\boldsymbol{p}_{k}=-\frac{\left|\boldsymbol{g}_{k}\right|^{2}}{\boldsymbol{g}_{k}^{T} \cdot \overline{\boldsymbol{H}}\left(\boldsymbol{x}_{k}^{m}\right) \cdot \boldsymbol{g}_{k}} \boldsymbol{g}_{k},
$$

where $\boldsymbol{g}_{k}=\nabla C\left(\boldsymbol{x}_{k}^{m}\right)=\operatorname{Re}\left\{\overline{\boldsymbol{J}}^{H}\left(\boldsymbol{x}_{k}^{m}\right) \cdot \boldsymbol{e}\left(\boldsymbol{x}_{k}^{m}\right)\right\}$ and $\overline{\boldsymbol{H}}\left(\boldsymbol{x}_{k}^{m}\right)=\nabla \nabla C\left(\boldsymbol{x}_{k}^{m}\right)=\operatorname{Re}\left\{\overline{\boldsymbol{J}}^{H}\left(\boldsymbol{x}_{k}^{m}\right) \cdot \overline{\boldsymbol{J}}\left(\boldsymbol{x}_{k}^{m}\right)\right\}$ are the first and second derivatives of the cost function at the $k$ th iteration. In these expressions $\overline{\boldsymbol{J}}$ is the $M \times 2$ Jacobian matrix and is given by the expression

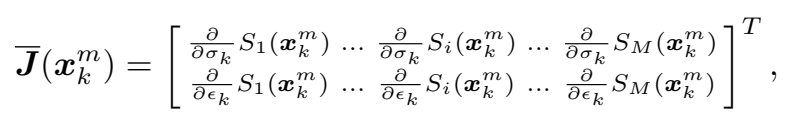

which contains the first derivatives of the measurements obtained from the initial-guess object with respect to the conductivity and permittivity. Using a similar numerical method as in [2], we can calculate (4) and update the object's electromagnetic properties. 


\section{NUMERICAL RESULTS}

In this section, we present the results of detecting the shape and material properties of a star-shaped object. The distances of this object's surface nodes from the origin range between $11 \mathrm{~mm}$ and $15 \mathrm{~mm}$. The relative permittivity and conductivity of the star-shaped object are 12 and $4 \mathrm{~S} / \mathrm{m}$, respectively. To collect the measurement data sets, we illuminate the object by 12 incident plane waves from six different directions (the $\pm x$, $\pm y$, and $\pm z$ directions) with both theta and phi polarizations. The phi and theta components of the scattered electric fields are measured from 26 directions in the far-field region and the operating frequency is $10 \mathrm{GHz}$.

The initial guess in this experiment is a sphere of radius $10 \mathrm{~mm}$ with a relative permittivity and conductivity of 6 and $6 \mathrm{~S} / \mathrm{m}$, respectively. In this experiment, we update the geometry of the initial-guess object in five iterations, and in the next five iterations, we update the material properties of the object. As we can see in Fig. 2, the initial-guess object reaches the general shape of the target in the first iterations, and the cost function in Fig. 1 decreases significantly in these iterations. The cost function finally drops to $4.9 \%$ of its initial value. As evident from Fig. 1, the exact values of the material properties of the target are obtained in 28 iterations.
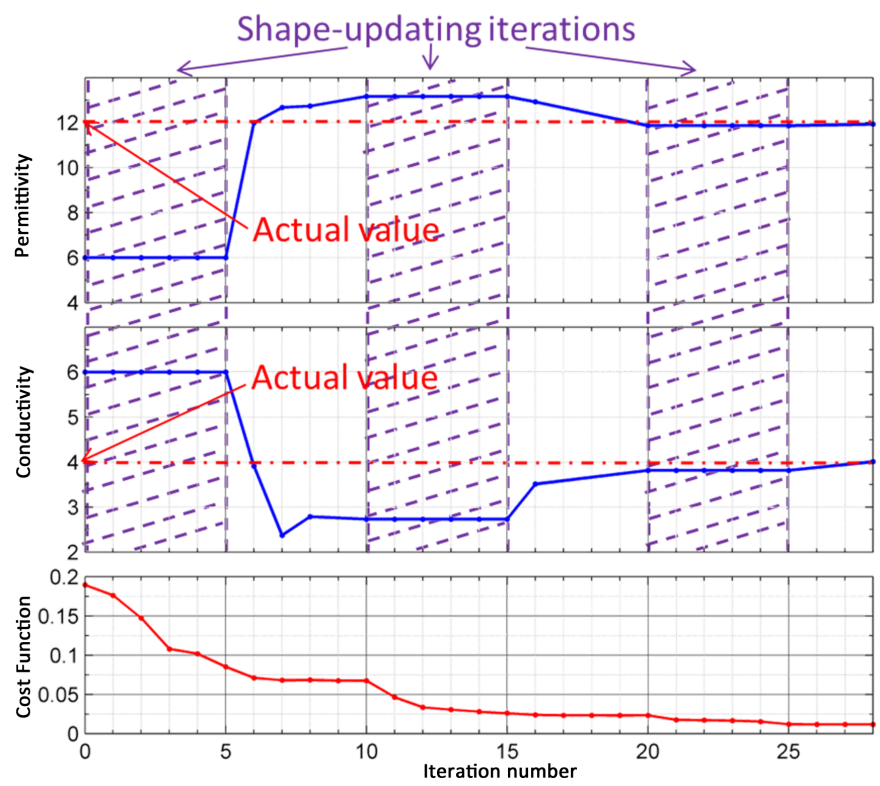

Fig. 1. Reconstruction of the permittivity and conductivity of a star-shaped target (the purple parts show the shape reconstruction iterations) and the cost function in 28 iterations.

\section{CONCLUSION}

The numerical results show the capability of our method in detecting the electromagnetic properties of unknown objects while we are reconstructing the shapes of these objects. The cost function decreases significantly in the first iterations, especially in the iterations where we update the geometry of

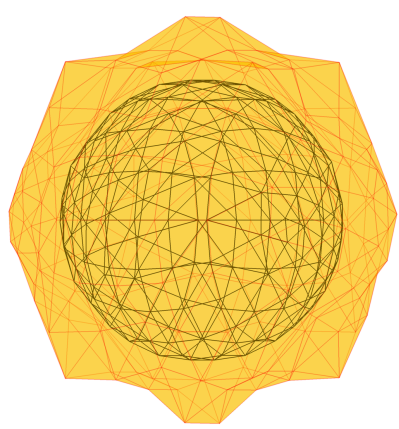

(a)

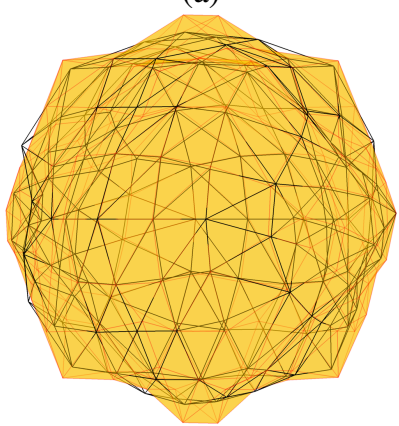

(c)

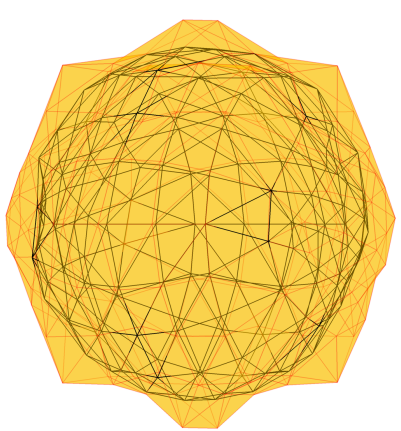

(b)

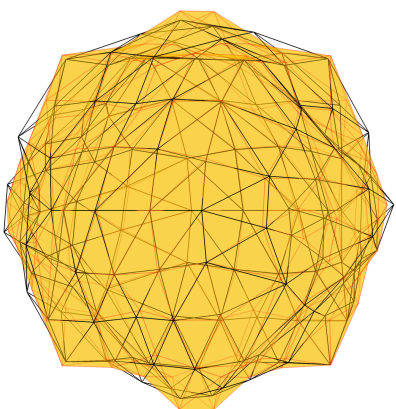

(d)
Fig. 2. Reconstruction of the star-shaped object at $10 \mathrm{GHz}$, where the yellow object is the target and the transparent object is the evolving object, in the (a) first iteration (initial guess), (b) fifth iteration, (c) fifteenth iteration, and (d) twenty-eighth iteration.

the initial-guess object, and finally the speed of reducing of the cost function slows down until it reaches zero.

\section{ACKNOWLEDGEMENT}

This work was supported by the Scientific and Technical Research Council of Turkey (TUBITAK) under Research Grant 111E203, by Schlumberger-Doll Research (SDR), and by contracts from ASELSAN, Turkish Aerospace Industries (TAI), and the Undersecretariat for Defense Industries (SSM).

\section{REFERENCES}

[1] S. C. Hagness, A. Taflove, and J. E. Bridges, "Three-dimensional FDTD analysis of a pulsed microwave confocal system for breast cancer detection: Design of an antenna-array element," IEEE Trans. Antennas Propagat., vol. 47, no. 5, pp. 783-791, 1999.

[2] A. Etminan and L. Gürel, "Microwave imaging of three-dimensional conducting objects using the Newton minimization approach," in Proc. Computational Electromagnetics Workshop, CEM'13, pp. 45-47, Izmir, Turkey, August 2013.

[3] A. Abubakar, T. M. Habashy, V. L. Druskin, L. Knizhnerman, and D. Alumbaugh, "A 2.5D forward and inverse modeling for interpreting low-frequency electromagnetic measurements," Geophysics, vol. 73, no. 4, pp. F165-F177, Aug. 2008.

[4] T. M. Habashy and A. Abubakar, "A general framework for constraint minimization for the inversion of electromagnetic measurements," Prog. Electromagn. Res., vol. 46, pp. 265-312, 2004.

[5] L. Gürel and Ö. Ergül, "Hierarchical parallelization of the multilevel fast multipole algorithm (MLFMA)," Proc. IEEE, vol. 101, pp. 332 341, 2013. 